\title{
General Conclusion
}

Quantitative pricing and investment are art forms which can profit from the exact sciences. These sciences are more than two hundred years old and show us the path to take as well as differences with the financial world. Financial equations can evolve with regulation and social behavior. However, central limit theorems establish some statistical attractors which are important invariants that should be captured. We present a methodology that combines statistical invariants with classical pricing models. We also show how martingale models, usually designed for pricing, can be used to assess the quality of an investment strategy. We define measurable quantities, that we call toxicity indices, which allow us to rank financial products and investment strategies.

We complement modeling tools by proposing risk-monitoring mechanisms to follow up on market regimes. We establish some essential properties needed to design the right signals to follow. We also present some powerful algorithms to filter these signals and extract a simple actionable output.

This book seeks to aid the general reader by offering an overall methodology to play with models and which take advantage of hindsight. The stance here is practical and provides techniques to build sound quantitative investment strategies and to test them properly. One main takeaway is that models are approximations of shifting reality. Contrary to the situation in physics, the relevance of financial models must be persistently monitored to ensure that they fit their end purpose. In pricing, they are designed to account for the cost of hedge while in investment they are designed to help predict the future and control risk. 